\title{
Periodontitis promotes the proliferation and suppresses the differentiation potential of human periodontal ligament stem cells
}

\author{
WEI ZHENG $^{1 *}$, SHI WANG ${ }^{2 *}$, JIANGUO WANG ${ }^{1}$ and FANG JIN ${ }^{3}$ \\ ${ }^{1}$ Department of Orthodontics, Tianjin Stomatological Hospital, Tianjin; ${ }^{2}$ Department of Stomatology, \\ The Third Central Hospital, Tianjin 300041; ${ }^{3}$ Department of Orthodontics, School of Stomatology, \\ Fourth Military Medical University, Xi'an, Shaanxi 710032, P.R. China
}

Received October 8, 2014; Accepted May 15, 2015

DOI: $10.3892 / \mathrm{ijmm} .2015 .2314$

\begin{abstract}
The aim of the present study was to investigate the periodontitis-associated changes in the number, proliferation and differentiation potential of human periodontal ligament stem cells (PDLSCs). Cultures of human periodontal ligament cells (PDLCs) were established from healthy donors and donors with periodontitis. The numbers of stem cell were characterized using flow cytometry. PDLSCs were isolated from the PDLCs by immunomagnetic bead selection. Colony-forming abilities, osteogenic and adipogenic potential, gene expression of cementoblast phenotype, alkaline phosphatase activity and in vivo differentiation capacities were then evaluated. Periodontitis caused an increase in the proliferation of PDLSCs and a decrease in the commitment to the osteoblast lineage. This is reflected by changes in the expression of osteoblast markers. When transplanted into immunocompromised mice, PDLSCs from the healthy donors exhibited the capacity to produce cementum PDL-like structures, whereas, the inflammatory PDLSCs transplants predominantly formed connective tissues. In conclusion, the data from the present study suggest that periodontitis affects the proliferation and differentiation potential of human PDLSCs in vitro and in vivo.
\end{abstract}

\section{Introduction}

Periodontitis is an inflammatory disease, which manifests clinically as a loss of connective periodontal tissues,

Correspondence to: Dr Jianguo Wang, Department of Orthodontics, Tianjin Stomatological Hospital, 75 Dagubei Road, Tianjin 300041, P.R. China

E-mail: wangjianguocn@hotmail.com

Dr Fang Jin, Department of Orthodontics, School of Stomatology, Fourth Military Medical University, 17 Changlexi Road, Xi'an, Shaanxi 710032, P.R. China

E-mail: jinfangkq@163.com

*Contributed equally

Key words: human, periodontitis, periodontal ligament stem cells, tissue engineering including periodontal ligament (PDL) and alveolar bone, resulting in loosening of the teeth, loss of chewing function and, ultimately, tooth loss $(1,2)$. To achieve successful periodontal regeneration, the formation of periodontal fibers, the insertion of these fibers into newly formed cementum and the restoration of alveolar bone height are required (3). To date, the restoration of damaged or diseased periodontal tissues is considered to be biologically possible, but clinically unpredictable, resulting in only partial regeneration at best (4).

The PDL is a highly fibrous and vascular tissue, which anchors the tooth root to the tooth socket bone and is important in tooth anchorage, sensation, facilitating nutrient delivery to surrounding cells and repairing damaged tissue in response to periodontal disease or mechanical trauma $(5,6)$. It has been demonstrated that human the PDL contains a heterogeneous population of cells, including cementoblasts, osteoblasts, fibroblasts, myofibroblasts, endothelial cells, nerve cells and epithelial cells $(1,5-7)$. However, the presence of paravascular progenitor stem cells within the PDL has long been hypothesized $(2,3,5,8)$, Seo et al (9) isolated PDL stem cells (PDLSCs) in the human PDL; PDLSCs exhibit mesenchymal stem cells characteristics, including clonogenicity and high proliferation, when transplanted into immunocompromised rodents. The PDLSCs demonstrated the capacity to generate a cementum/PDL-like structure and contribute to periodontal tissue repair. Therefore the application of PDL stem cells may hold promise as a therapeutic approach for the reconstruction of tissues destroyed by periodontal diseases (10).

To investigate this potential, a thorough understanding of PDLSCs is required. Whether inflammatory changes in the periodontium can affect stem cells remains to be elucidated. A full understanding of the properties of stem cells from periodontitis-affected teeth is required prior to them being used to treat periodontal disease. In the present study, the effects of periodontitis on the proliferation and differentiation of PDLSCs were investigated through in vivo and in vitro experiments. The aim of the present study was to provide a better understanding of the mechanism of periodontitis, and offer insight into the selection of candidate cells for periodontal regeneration between healthy and periodontitis-affected PDLSCs. 


\section{Materials and methods}

Patients. Disease free premolars and third molars were collected from 10 patients, following extraction for orthodontic treatment purposes, aged between 30 and 40 years old. Diseased teeth were obtained from 10 patients with generalized chronic periodontitis, undergoing tooth extraction, also aged between 30 and 40 years of age. The clinical diagnosis of chronic periodontal disease was based on visual and radiographic assessment of the periodontal tissues, and on measurements of the space between the tooth and gum. These spaces are normally $1-3 \mathrm{~mm}$ in depth, and deepen as supporting connective tissue and bone are lost (11). All selected patients presented with generalized chronic periodontitis with more than one pocket depth $\geq 5 \mathrm{~mm}$. All subjects were free of any clinical evidence of recent infection. The subjects included in the study had never smoked, had no history of systemic disease, had received no periodontal treatment, had not received antibiotics, anti-inflammatory drugs or any other drugs in the pervious 6 months, and were not alcohol or antioxidant vitamin consumers. Individuals living in this region consume a diet rich in fish, vegetables and fruit due to the conditions in the region and traditional lifestyles. This study was approved by the Ethics Committee of the Tianjin Stomatological Hospital (Tianjin, China). Written informed consent was provided by all participants prior to enrollment.

Cell culture. Primary cultures from the two groups were obtained by culturing explants of the healthy and periodontitis-affected periodontal tissues from patients undergoing extractions. The PDL tissue was gently scraped from the surface of the middle part of the root, minced into $1-\mathrm{mm}^{3}$ cubes and placed into 6-well culture dishes (Costar, Cambridge, MA, USA). The explants were grown in a-minimal essential medium (MEM) supplemented with $10 \%$ fetal bovine serum (FBS), $0.292 \mathrm{mg} / \mathrm{ml}$ glutamine, $100 \mathrm{U} / \mathrm{ml}$ penicillin/streptomycin and $100 \mu \mathrm{M} / 1$ ascorbic acid. The cultures were incubated at $37^{\circ} \mathrm{C}$ in a humidified atmosphere of $5 \% \mathrm{CO}_{2}$.

Flow cytometric analysis. Cell-surface antigen expression was performed using flow cytometric analysis; $1 \times 10^{6}$ PDLCs were washed in PBS, and then incubated with mouse anti-human monoclonal STRO-1 (dilution 1:100; R\&D Systems, Inc., Minneapolis, MN, USA) antibodies for $30 \mathrm{~min}$ at $4^{\circ} \mathrm{C}$. The cells were washed twice with cold phosphate-buffered saline (PBS) containing $2 \% \mathrm{FBS}$, and incubated with $1 \mu \mathrm{g}$ fluorescein isothiocyanate (FITC)-conjugated goat anti-mouse IgM antibodies (Santa Cruz Biotecgnology, Inc., Santa Cruz, CA, USA) for $30 \mathrm{~min}$ at $4^{\circ} \mathrm{C}$. Mouse isotype antibodies (BD Biosciences, Franklin Lakes, NJ, USA) served as a control. The labeled cells were analyzed using a flow cytometer (Cell Lab Quanta; Beckman Coulter, Fullerton, CA, USA).

Isolation of PDLSCs. To obtain STRO- $1^{+}$stem cells, the two cell populations were sorted using immunomagnetic beads (Dynabeads; Dynal Biotech, Oslo, Norway), according to the manufacturer's instructions. The cells $\left(\sim 4 \times 10^{6}\right)$ were incubated with mouse anti-human STRO-1 supernatant (R\&D Systems) for $30 \mathrm{~min}$ at $4^{\circ} \mathrm{C}$. These cells were then washed with $\mathrm{PBS} / 5 \% \mathrm{FBS}$ and re-suspended with rat anti-mouse IgM- conjugated Dynabeads (four beads per cell) for 60 min using a rotary mixer. Following washing, the bead-positive cells were segregated using a magnetic particle separator and subsequently seeded into $75-\mathrm{cm}^{2}$ culture flasks (Costar) at $37^{\circ} \mathrm{C}$ in $5 \% \mathrm{CO}_{2}$.

Colony-forming unit-fibroblast assay. Colony-forming assays were performed using the PDLSCs, which were plated at a density of between 0.25 and $1 \times 10^{4} /$ well in a 6 -well plate. Following 10 days of culture, the two cultures were fixed with $4 \%$ buffered formaldehyde and then stained with $0.1 \%$ toluidine blue (Sigma-Aldrich, St. Louis, MO, USA). The cells were visualized using a Cambridge 360 Scanning Electron Microscope (Carl Zeiss SMT, Thornwood, NY, USA). Aggregates of $>50$ cells were scored as colonies.

Osteogenic and adipogenic differentiation. For osteogenesis, the PDLSCs were plated at a density of $5 \times 10^{3} \mathrm{~cm}^{2}$ in 24-well plates and cultured for 3 days. The cells were then incubated with a-MEM, supplemented with $10 \% \mathrm{FBS}, 50 \mu \mathrm{M}$ L-ascorbic acid 2-phosphate (Sigma-Aldrich), $10 \mathrm{mM} \beta$-glycerophosphate (Sigma-Aldrich) and $100 \mathrm{nM}$ dexamethasone (Sigma-Aldrich), for 2 weeks to induce mineral formation. The cells were fixed with $70 \%$ ethanol for 15 min and stained with $2 \%$ alizarin red ( $\mathrm{pH} 4.0$; Sigma-Aldrich) for $15 \mathrm{~min}$. The nodule area was measured quantitatively using an image analysis system (Image-Pro Plus 5.0; Media Cybernetics, Inc., Baltimore, MD, USA).

For adipogenesis, the PDLSCs were plated at a density of $5 \times 10^{3} \mathrm{~cm}^{2}$ in 24-well plates and were cultured for 3 days. The cells were then incubated with a-MEM, supplemented with $10 \%$ FBS, $0.5 \mathrm{mM}$ methylisobutylxantine, $0.5 \mu \mathrm{M}$ hydrocortisone and $60 \mu \mathrm{M}$ indomethacin (Sigma-Aldrich). The cells were cultured for an additional 21 days. The adipogenic cultures were fixed in $70 \%$ ethanol for 15 min and stained with $2 \%$ fresh Oil Red O solution (Sigma-Aldrich) for $15 \mathrm{~min}$. The lipid area was measured quantitatively using an image analysis system (Image-Pro Plus 5.0; Media Cybernetics, Inc.).

Reverse transcription-quantitative polymerase chain reaction $(R T-q P C R)$. PDLSCs at $90 \%$ confluence were harvested. The total celluar RNA was isolated from the maintenance cell cultures using TRIzol reagent (Invitrogen Life Technologies, Carlsbad, CA, USA). RT-PCR was performed with $1 \mathrm{mg}$ RNA using a PrimeScript RT reagent kit (Takara, Dalian, China). The RNA concentration was calculated using a Nanodrop ND2000 spectrophotometer (Nano-Drop Technologies, Wilmington, DE, USA). qPCR was performed using $1 \mu \mathrm{g}$ cDNA (12). First-strand cDNA syntheses were performed, as reported previously (12). For qPCR a QuantiTect SYBR-Green kit (Toyobo, Osaka, Japan) and ABI Prism 7700 Sequence Detection System (Applied Biosystems, Foster City, CA, USA) were used. Primer sequences for Runx2 (GenBank accession no. NM 004348), collagen type I (GenBank accession no. NM 000088), osteocalcin (GenBank accession no. NM 000711), were as follows: Runx2, sense 5'-CCCGTGGCCTTCAAGGT-3' and antisense 5'-CGTTACCCGCCATGACAGTA-3'; collagen type I, sense 5'-CCAGAAGAACTGGTACATCAGCAA-3' and antisense 5'-CGCCATACTCGAACTGGAATC-3'; and osteocalcin, sense 5'-AGCAAAGGTGCAGCCTTTGT-3' and antisense 5'-GCGCCTGGGTCTCTTCACT-3'. The gene expression 
of GAPDH was used as a reference gene in all applications. The qPCR eactions were performed using the following cycling conditions: $95^{\circ} \mathrm{C}$ for $10 \mathrm{~min}$, then 45 cycles of $95^{\circ} \mathrm{C}$ for $15 \mathrm{sec}$ followed by $60^{\circ} \mathrm{C}$ for $1 \mathrm{~min}$.

Alkaline phosphatase (ALP) activity assay. At defined time-points, the activity of ALP in the PDLSCs was detected using an ALP assay kit (Nanjing Jiancheng Bioengineering Institute, Nanjing, China). In brief, the cells ( $1 \times 10^{3} /$ well) were seeded into a 96-well plate. Following culture, cells were washed three times with $0.01 \mathrm{M}$ PBS and $50 \mathrm{ml}$ cold $10 \mathrm{mM}$ Tris $/ \mathrm{HCl}$ buffer (pH 7.4; Sigma-Aldrich) containing 0.1\% Triton X-100 (Sigma-Aldrich) added prior to incubation at $4^{\circ} \mathrm{C}$ overnight. Subsequently, $100 \mu \mathrm{l}$ ALP substrate solution $\left(2 \mathrm{mM} \mathrm{MgCl}_{2}\right.$ and $16 \mathrm{mM}$ p-nitrophenyl phosphate; Sigma-Aldrich) was mixed with each sample. Following incubation at $37^{\circ} \mathrm{C}$ for $30 \mathrm{~min}$, the reaction was terminated by the addition of $50 \mathrm{ml} 0.2 \mathrm{M} \mathrm{NaOH}$. The optical density values were measured at $405 \mathrm{~nm}$ in a spectrophotometer using a microplate reader (DU 640).

In vivo differentiation assay. For a single in vivo transplantation, $2 \times 10^{6}$ PDLSCs were mixed with $20 \mathrm{mg}$ ceramic bovine bone (CBB; Research and Development Center for Tissue Engineering, Fourth Military Medical University, Xi'an, China), powders and the mixture was centrifuged briefly at 1,300 rpm at room temperature for $3 \mathrm{~min}$ and the supernatant was discarded. The pelleted powder with adherent cells was mixed with $15 \mu 1$ Matrigel (Beixiu, Guangzhou, China) to form a clot, which was implanted into subcutaneous pockets on the dorsal surface of 4-week-old NOD/SCID mice (weighing $20 \mathrm{~g}$ ). The animals were divided into 2 groups, 15 rats in each group. The animals were housed in filter top cages (at $26^{\circ} \mathrm{C}$ ) and were given a standard diet and water with antibiotics and antimycotics ad libitum. All animal use protocols were reviewed and approved by the Animal Care Committee of Fourth Military Medical University. The implants were recovered after 8 weeks, fixed in $4 \%$ paraformaldehyde for 2 days, and then decalcified for a further 10 days in 10\% EDTA (Sigma-Aldrich) prior to embedding in paraffin. For histological analysis, $5 \mu \mathrm{m}$ sections of the implants were prepared and stained using hematoxylin and eosin (H\&E).

Statistical analysis. Statistical significance was assessed using a $\chi^{2}$ test and an independent samples t-test with SPSS v.12.0 software (SPSS, Inc., San Rafael, CA, USA). P $<0.05$ was considered to indicate a statistically significant difference. All data acquisition and analyses were performed in a blinded-manner.

\section{Results}

Radiographic features of periodontal tissues. The clinical diagnosis of the periodontal disease was based on radiographic assessment of the periodontal tissues, as shown in Fig. 1.

Effect of periodontitis on the number of PDLSCs. FACS analysis of the PDL cells demonstrated that the percentage positivity for STRO-1 in the periodontitis-affected PDL cells was significantly higher than those in the healthy PDL cells. The rate of STRO $-1^{+}$cells was $18.2 \pm 3.2 \%$ in the healthy donors and $50.3 \pm 5.1 \%$ in the periodontitis-affected donors $(\mathrm{P}<0.05$; Fig. 2).

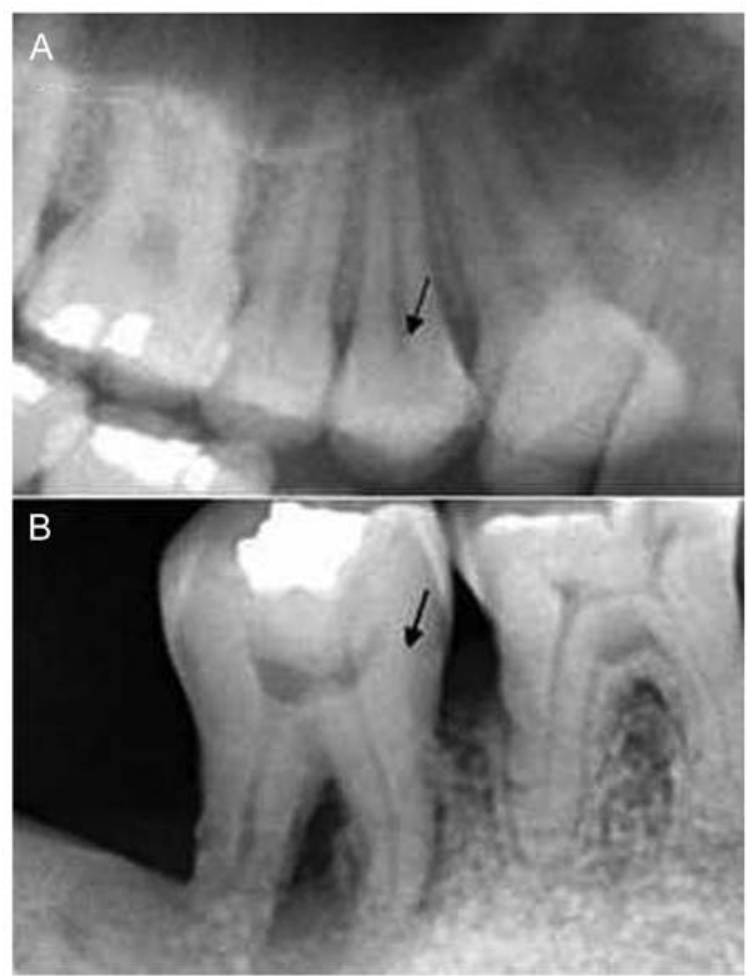

Figure 1. Intra-oral dental radiograph of the patients. (A) Radiograph from a healthy donor undergoing premolar extractions for orthodontic treatment purposes. (B) Radiograph of mandibular second molar from a periodontitis-affected donor confirms severe periodontal loss and a large bony defect associated with the periodontal disease. The arrows indicate the extracted teeth.

Effect of periodontitis on the proliferation of PDLSCs. The ability of colony forming was compared between healthy PDLSCs and periodontitis-affected PDLSCs. The cells in the two groups demonstrated the ability to form multi-cell clusters (Fig. 3A and B). In the PDLSCs from the healthy donors, the formation of $\sim 30$ colonies, generated from single cells cultured at low density for 10 days, was observed; whereas the PDLSCs from the periodontitis-affected donors generated 50 colonies. These results revealed a statistically significant difference, with the PDLSCs from the periodontitis-affected donors having increased colony forming abilities, compared with the healthy PDLSCs (Fig. 3C).

Effect of periodontitis on the in vitro pluripotential capacity of PDLSCs. During osteogenic stimulation, the appearance of undifferentiated PDLSCs change from their characteristic spindle-shaped morphology to a cuboidal appearance. The deposition of calcified matrix on the culture dishes was assessed by the quantity of alizarin red staining. A decline in the quantity of mineralized matrix formed was observed in the diseased PDLSC culture (Fig. 4A and B).

As adipogenesis progressed, the accumulation of large cytoplasmatic vacuoles containing lipids was detected using Oil Red staining, and were visualized under phase-contrast microscopy. The adipocytes, which developed in the cultures derived from healthy donors accumulated more fat than the adipocytes, which developed in the cultures derived from periodontitis-affected donors (Fig. 4C and D). A signifi- 
A

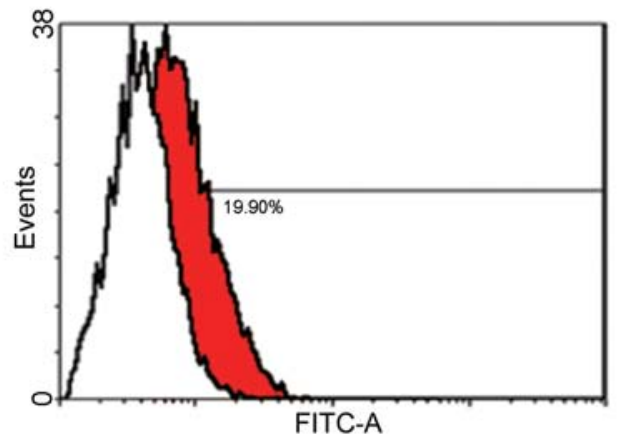

B

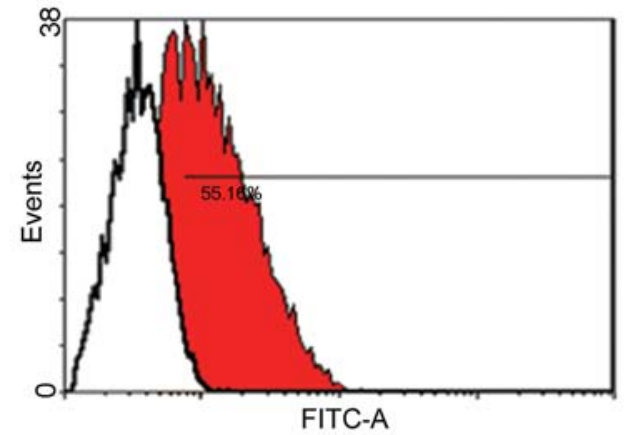

C

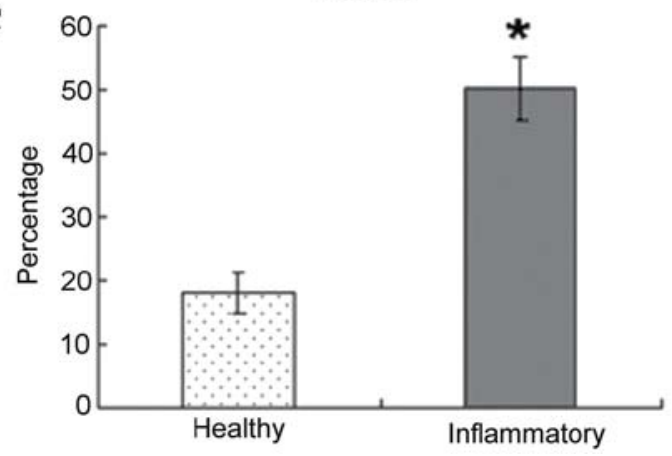

Figure 2. Periodontitis-associated changes in the number of PDLSCs. (A) Flow cytometry data of PDLCs from a healthy donor. (B) Flow cytometry data of PDLCs from a periodontitis-affected donor. (A and B) Red areas indicate STRO1-positive cells, white areas indicate STRO-1-negative cells. (C) Numbers of PDL-derived STRO-1 ${ }^{+}$cells were significantly increased in the inflammatory group, compared with the healthy group $\left({ }^{*} \mathrm{P}<0.05\right)$. PDLC, periodontal ligament cell; FITC, fluorescein isothiocyanate.

cant difference was observed in the in vitro A significant difference was observed in the in vitro osteogenic activity $(\mathrm{P}<0.05 ;$ Fig. 4E).and adipogenic activity in the two cell groups $(\mathrm{P}<0.05 ;$ Fig. 4F).

Effect of periodontitis on gene expression and ALP activity in PDLSCs. The present study used RT-qPCR to examine whether changes in the differentiation potential of the PDLSCs were accompanied by changes in the expression of phenotype-specific gene markers and ALP activity. In basal, non-differentiating conditions, the PDLSCs derived from periodontitis-affected donors expressed lower mRNA levels of the osteoblast markers, Runx2, collagen 1 and osteocalcin, compared with the PDLSCs isolated from healthy donors (Fig. 5A). The PDLSCs from healthy donors exhibited higher ALP reactivity, compared with the PDLSCs from periodontitis-affected donors between days 3 and 14 of the observation period (Fig. 5B).
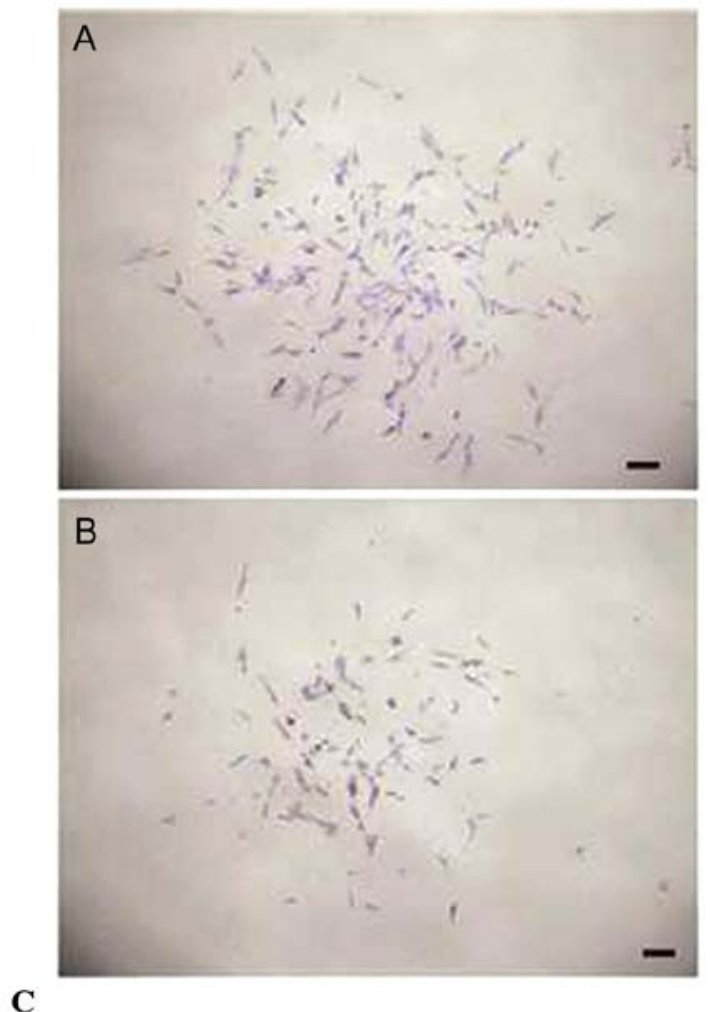

C

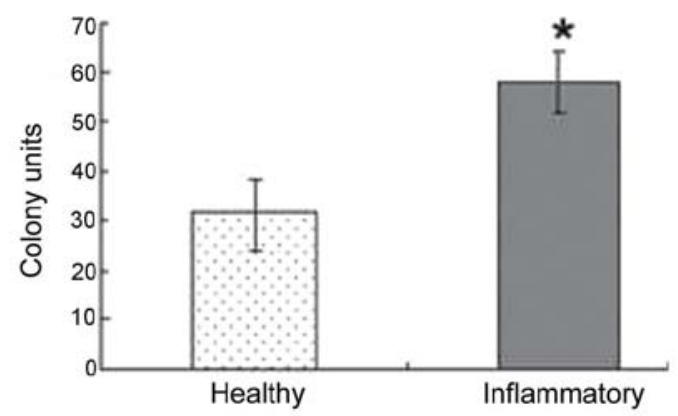

Figure 3. PDLSC colony formation assay. Primary colony formation by (A) PDLSCs from healthy and (B) periodontitis-affected donors. (C) Graph indicates a statistically significant difference in the total number of colonies between the two culture groups. Values are expressed as the mean \pm standard deviation $\left({ }^{*} \mathrm{P}<0.05\right)$. Scale bar $=100 \mu \mathrm{m}$. PDLSC, periodontal ligament stem cell.

Effect of periodontitis on the in vivo differentiation of PDLSCs. The PDLSC transplants from the healthy donors were observed to posses the tissue-regenerative capacity to generate cementum-like mineralized tissue on the border of the CBB, with PDL-like fibrous tissue interfacing these areas (Fig. 6A and B) and cementocyte-like cells embedded in the mineralized structure (Fig. 6C; arrows). By contrast, the implants containing periodontitis-affected PDLSCs formed small quantities of mineralized tissue lining the CBB surfaces (Fig. 6D and E); and fibrous tissues were observed within the CBB only (Fig. 6F).

\section{Discussion}

For decades periodontists have investigated ways to repair the damage that occurs during periodontitis. This has included the use of a range of surgical procedures and a variety of grafting materials. Due to issues of safety, effec- 

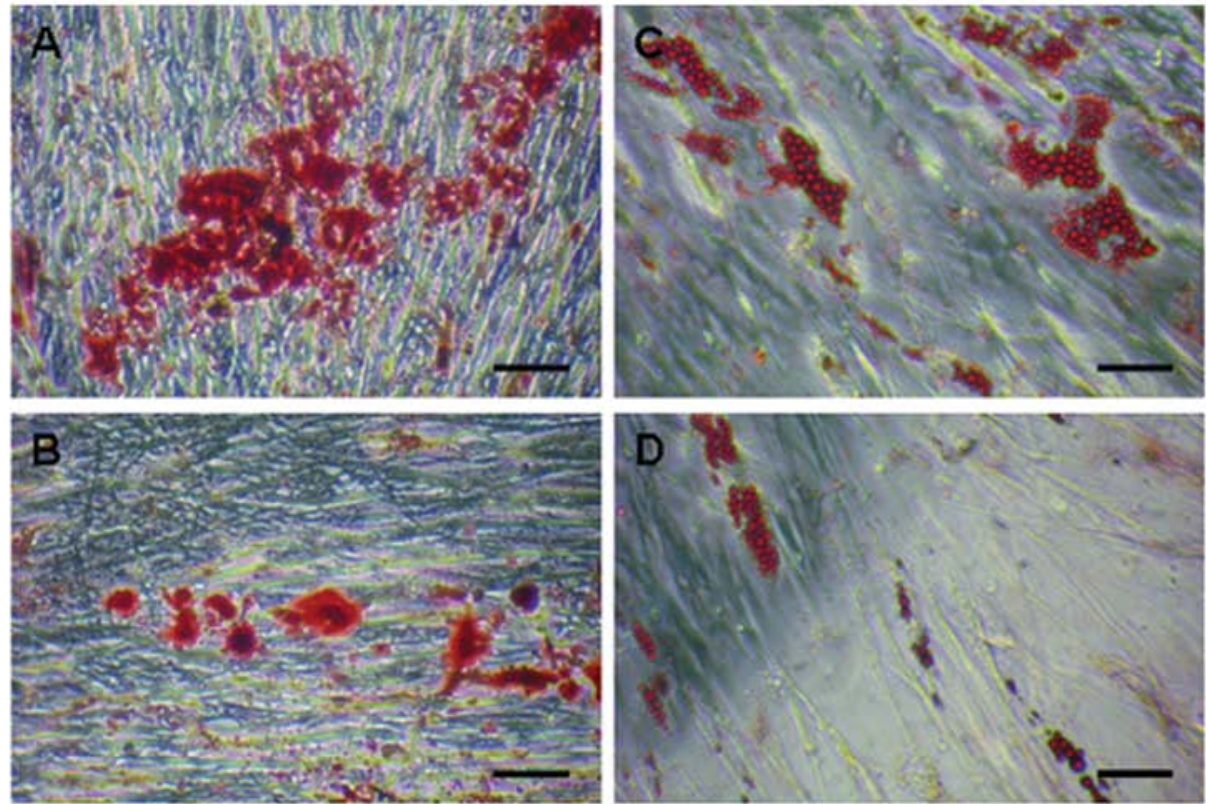

$\mathbf{E}$

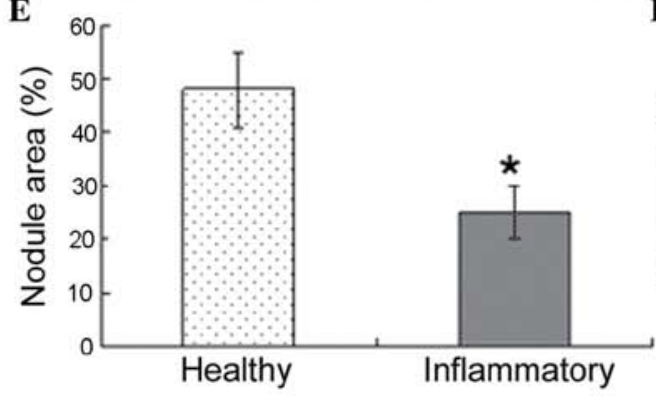

F

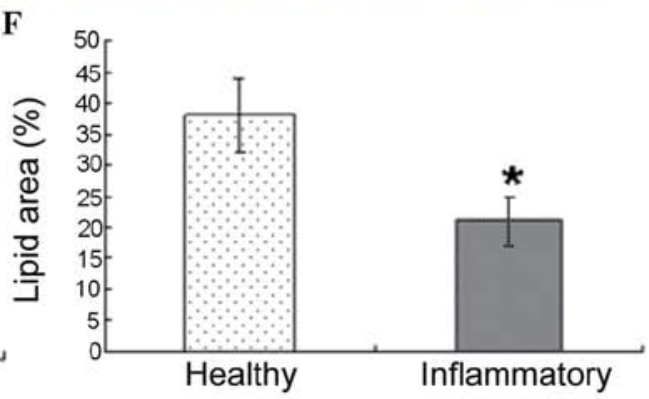

Figure 4. Multipotent differentiation of periodontal ligament stem cells. Representative images of alizarin red staining of osteocytes from (A) healthy and (B) periodontitis-affected donors. Representative images of Oil Red O-staining of adipocytes from (C) healthy and (D) periodontitis-affected donors. Correlations between the two cell groups and (A) osteogenic and (B) adipogenic potential, respectively $\left({ }^{*} \mathrm{P}<0.05\right)$. Scale bar $=100 \mu \mathrm{m}$.

A

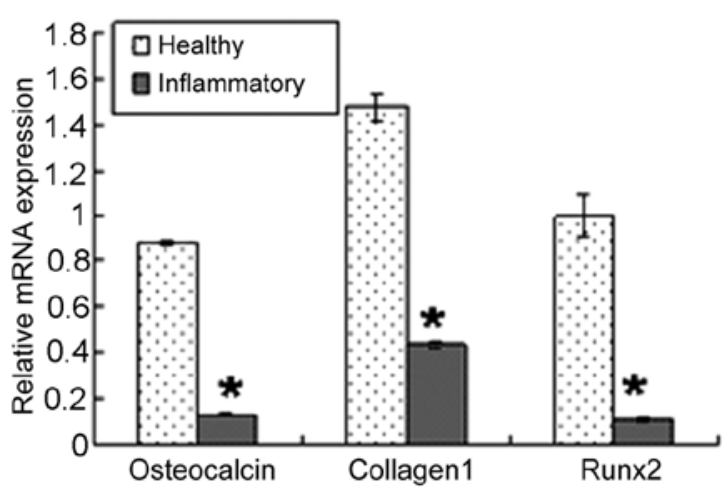

B

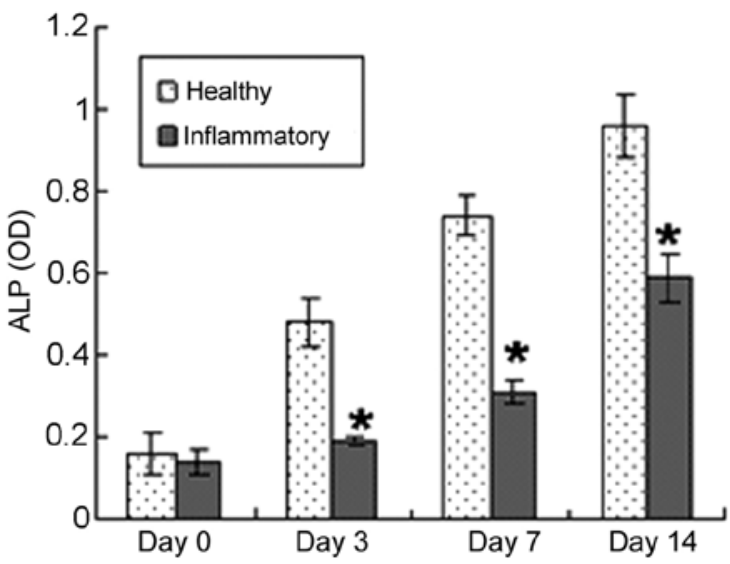

Figure 5. Gene expression of osteoblast markers in PDLSCs. (A) Gene expression levels of osteoblast markers in PDLSCs between healthy and periodontitis-affected donors was determined using reverse transcription-quantitative polymerase chain reaction. The expression values were normalized to the expression of GAPDH ( $\left.{ }^{*} \mathrm{P}<0.05\right)$. (B) Comparison in ALP activity between healthy and periodontitis-affected PDLSCs ("P<0.05). PDLSC, periodontal ligament stem cell; ALP, alkaline phosphatase; OD, optical density.

tiveness and stability, their use for periodontal regeneration has been questioned $(2,4,13)$. Biological approaches based on the principles of tissue-engineering have emerged as prospective alternatives. Therefore, PDLSCs offer potential for the development of novel cell-based therapies to treat the damage caused by trauma or periodontal disease $(2,6,14)$. Whether PDLSCs from periodontitis-affected teeth can be used to regenerate periodontal tissues remains to be 

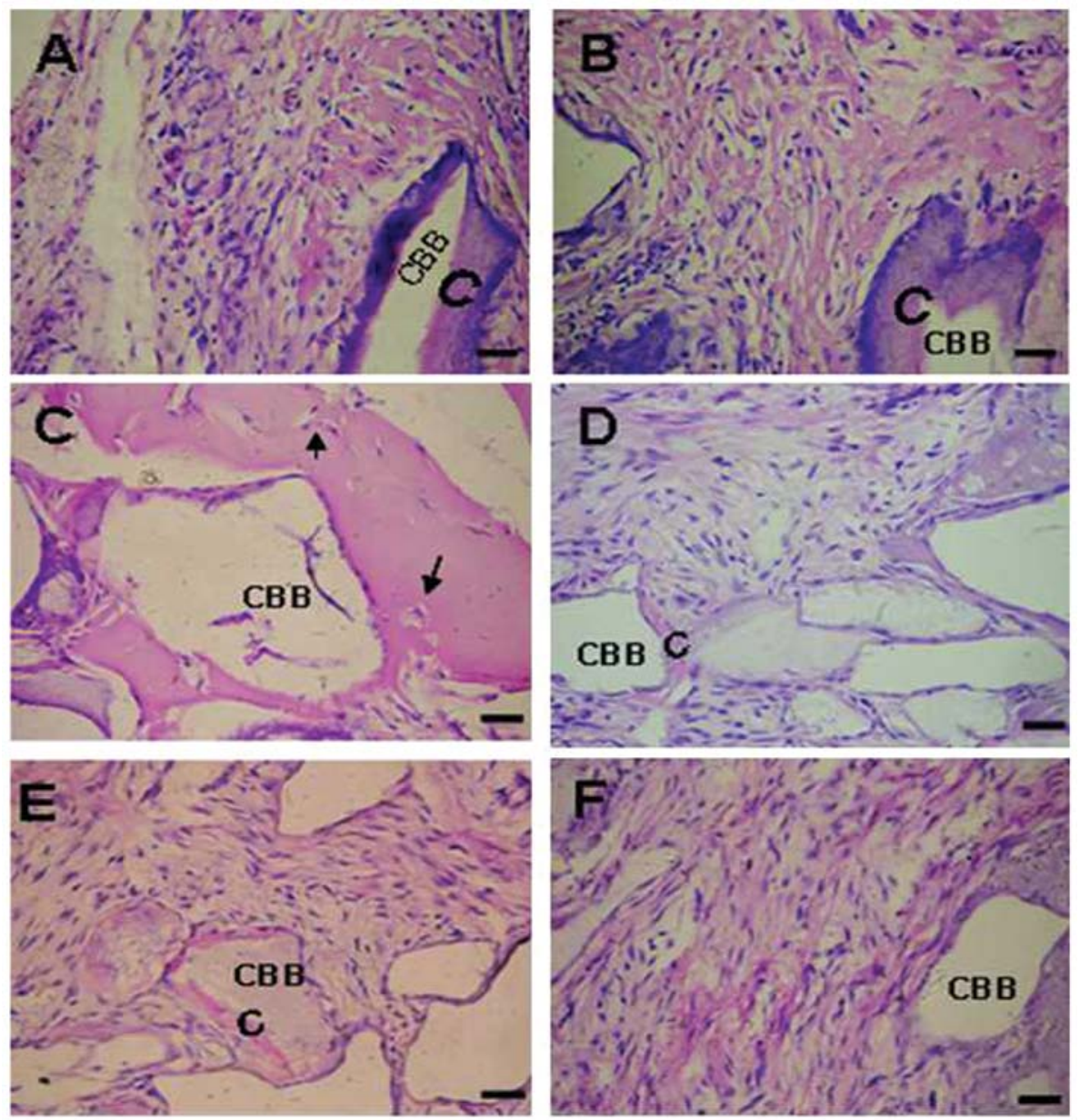

Figure 6. Differentiation potential of PDLSCs in vivo. (A-C) Transplants from healthy PDLSCs. (D-F) Transplants from periodontitis-affected PDLSCs. Arrows indicate cementocyte-like cells embedded in the mineralized structure. Scale bar $=100 \mu \mathrm{m}$. CBB, ceramic bovine bone; C, cementum-like mineralized tissue.

elucidated. Previous investigation of the in vitro and in vivo features of stem cells from periodontitis-affected PDL has been limited, and investigation of the changes in the proliferation and differentiation potentials of PDLSCs when extracted from healthy and periodontitis-affected donors is of significant interest. Therefore, the present study investigated for the first time, to the best of our knowledge, the association between periodontitis and PDL stem cells.

In the present study, PDLSCs were successfully isolated and characterized from periodontitis-affected donors. Previous studies have demonstrated that the putative stem cell marker, STRO-1, which is used to isolate and purify bone marrow stromal stem cells, is also expressed by human PDLSCs. Thus, its expression can be used to isolate and characterize human PDLSCs $(3,6)$. The present study demonstrated that the percentage of positivity for STRO-1 from periodontitis-affected PDL cells was significantly higher than that in the healthy PDL cells. The effect of periodontitis on the number of stem cells revealed in the present study was also in agreement with previous data that wider distributions of stem cells are detected in sections of diseased ligaments (15). The increase in stem cell populations may be caused by the early recruitment and migration of paravascular cells, as has been observed in the wounded mice PDL $(15,16)$. In the present study, the clonogenic capabilities of PDLSCs from the periodontitis-affected donors were higher, compared with those from the healthy donors. Digirolamo et al (17) reported that the replicative potential of cells in culture is determined best using a simple colony-forming assay, and the samples with the high colony forming efficiency exhibit the highest replicative potential. In the present study, marked differences in the growth pattern of PDLSCs obtained from the healthy and periodontitis-affected donors were observed. This suggested that periodontitis had a significant effect on PDLSCs, in terms of proliferative activity. One possibility may be that periodontitis-affected tissue-derived stem cells exhibit higher mitotic properties, compared with cells derived from healthy donors.

In addition to the effect of periodontitis on PDLSC numbers and proliferation, the present study investigated its effects on function, in which the PDLSCs were characterized by their capacity to differentiate into various stromal cell lineages in vitro and in vivo. In the presence of osteogenic differentiation medium, the PDLSCs from healthy donors exhibited a higher alizarin-red-positive area, compared with the PDLSCs from periodontitis-affected donors. This result indicated increased osteogenic characteristics in the healthy PDLSCs. These results were supported by the gene expression profiles, determined using RT-qPCR analyses. The RT-qPCR results revealed periodontitis-associated PDLSC declines in the expression of Runx2, the earliest differentiation marker for 
committed osteogenetic precursor cells (18), and the expression of collagen and osteocalcin osteoblast markers (19). Analysis of these osteoblast markers revealed that the healthy PDLSCs had higher inherent differentiation, compared with the diseased PDLSCs. ALP is vital in calcified tissue formation by regulating phosphate transfer (20). In the present study, the higher ALP activity and expression of cementoblast phenotype suggested that PDLSCs from healthy donors may be more likely to differentiate into osteoblast/cementoblast-like cells. In addition to their osteogenic differentiation capacity, PDLSCs can differentiate into adipocytes, and thus, adipocyte differentiation has been used as a marker for the multipotential nature of the cells (21). In the present study, the periodontitis-affected PDLSCs formed fewer adipocytes than the healthy PDLSCs. These findings suggested that periodontitis reduced the pluripotential capacity of the PDLSCs. The present study performed an in vivo tissue regeneration assay, based on subcutaneous implantation of PDLSCs into a mouse transplantation model. The findings demonstrated that periodontitis decreased the ability of the PDLSCs to form periodontal tissues in vivo, which was in accordance with the previous suggestion that proliferation and differentiation are inversely correlated with each other due to the existence of dual-function regulators involved in controlling the two processes $(22,23)$. Periodontitis-affected individuals generally have an impaired ability to regenerate tissues (11). The present findings suggested that this impairment may be due to the decrease in stem cell differentiation ability. Periodontitis-associated defects in PDL function may be due to quantitative stem cell defects and, whether the cellular mechanism may be associated with the accumulation of growth factors and cytokines, including fibroblast growth factor, platelet-derived growth factor, insulin-like growth factor, transforming growth factor, tumor necrosis factor- $\alpha$, interleukin (IL)-l $\alpha$, IL-1 $\beta$, IL- 6 and IL-12, merit further investigation. Previous studies have demonstrated that the excessive and/or continuous production of cytokines in inflamed periodontal tissues may be responsible for the progression of periodontitis and periodontal tissue destruction $(24,25)$. As previously reported, a complex network of growth factors and cytokines guides cellular differentiation and regeneration in all organs and tissues (26). Therefore, the results of the present study demonstrated that certain components of inflammatory cytokines act, not only as promoters for cell proliferation, but also as inhibitors for PDLSC differentiation. The impaired regenerative potential of inflamed PDLSCs may be improved by a decrease or dilution of inflammatory cytokines.

As the development of novel medical therapies that use adult stem cell transplants to cure, repair or even grow a new organ (19) progresses, it is of important clinical significance to clarify the biological characteristics and functional changes of PDLSCs in periodontitis-affected individuals, to guide the treatment of periodontal disease. In conclusion, the present study demonstrated that, during periodontitis, the status of PDLSCs change with respect to their proliferation and intrinsic differentiation potential. The present study revealed that the decrease in the differentiation capacity of PDLSCs from periodontitis-affected donors may result in the decrease of periodontal tissue forming ability. In addition, the findings suggested that treatment, which increases the differentiation potential of PDLSCs is an important aim in developing novel therapeutic interventions to treat periodontal disease. If PDLSCs emerge as a candidate cell source for periodontal engineering, healthy PDL is a preferable source. If they are from diseased teeth, PDLSCs may commit to periodontal tissues by modulating cytokines and/or other differentiation strategies. Therefore, the therapeutic potential of healthy and inflammatory PDLSCs differs, which is important to consider when these cells are considered for therapy.

\section{Acknowledgements}

The present study was supported by grants from the Nature Science Foundation of China (grant. nos. 31030033 and 81171001). The authors would like to thank Dr Lei Wang and Dr Huan Shen (both from the School of Stomatology, Fourth Military Medical University) for their assistance with animal experiments.

\section{References}

1. Gronthos S, Mrozik K, Shi S and Bartold PM: Ovine periodontal ligament stem cells: isolation, characterization, and differentiation potential. Calcif Tissue Int 79: 310-317, 2006.

2. Lin NH, Gronthos S and Bartold PMS: Stem cells and periodontal regeneration 2000. Aust Dent J 53: 108-121, 2008.

3. Ivanovski S, Gronthos S, Shi S and Bartold PM: Stem cells in the periodontal ligament. Oral Dis 12: 358-363, 2006.

4. Benatti BB, Silvério KG, Casati MZ, Sallum EA and Nociti FH Jr: Physiological features of periodontal regeneration and approaches for periodontal tissue engineering utilizing periodontal ligament cells. J Biosci Bioeng 103: 1-6, 2007.

5. Ibi M, Ishisaki A, Yamamoto M, et al: Establishment of cell lines that exhibit pluripotency from miniature swine periodontal ligaments. Arch Oral Biol 52: 1002-1008, 2007.

6. Bartold PM, Xiao Y, Lyngstaadas SP, Paine ML and Snead ML: Stem cells and periodontal regeneration. Periodontol 40: 164-172, 2006.

7. Tomokiyo A, Maeda H, Fujii S, Wada N, Shima K and Akamine A: Development of a multipotent clonal human periodontal ligament cell line. Differentiation 76: 337-347, 2008.

8. Nagatomo K, Komaki M, Sekiya I, Sakaguchi Y, Noguchi K, Oda S, Muneta T and Ishikawa I: Stem cell properties of human periodontal ligament cells. J Periodont Res 41: 303-310, 2006.

9. Seo BM, Miura M, Gronthos S, Bartold PM, Batouli S, Brahim J, Young M, Robey PG, Wang CY and Shi S: Investigation of multipotent postnatal stem cells from human periodontal ligament. Lancet 364: 149-155, 2004.

10. Liu Y, Zheng Y, Ding G, Fang D, Zhang C, Bartold PM, Gronthos S, Shi S and Wang S: Periodontal ligament stem cell-mediated treatment for periodontitis in miniature swine. Stem Cells 26: 1065-1073, 2008.

11. Pihlstrom BL, Michalowicz BS and Johnson NW: Periodontal diseases. Lancet 366: 1809-1820, 2005.

12. Lindroos B, Mäenpää K, Ylikomi T, Oja H, Suuronen R and Miettinen S: Characterisation of human dental stem cells and buccal mucosa fibroblasts. Biochem Biophys Res Commun 368: 329-335, 2008.

13. Bartold PM, Xiao Y, Lyngstaadas SP, Paine ML and Snead ML: Principles and applications of cell delivery systems for periodontal regeneration. Periodontol 41: 123-135, 2006.

14. Sonoyama W, Liu Y, Fang D, et al: Mesenchymal stem cell-mediated functional tooth regeneration in swine. PLoS One 1: e79, 2006.

15. Chen SC, Marino V, Gronthos S and Bartold PM: Location of putative stem cells in human periodontal ligament. J Periodont Res 41: 547-553, 2006.

16. McCulloch CA and Melcher AH: Cell density and cell generation in the periodontal ligament of mice. Am J Anat 167: 43-58, 1983.

17. Digirolamo CM, Stokes D and Colter D: Propagation and senescence of human marrow stromal cells in culture: a simple colony-forming assay identifies samples. with the greatest potential to propagate and differentiate. Br J Haematol 107: 275-281, 1999. 
18. Fakhry A, Ratisoontorn C, Vedhachalam C, et al: Effects of FGF-2/-9 in calvarial bone cell cultures: differentiation stage-dependent mitogenic effect, inverse regulation of BMP-2 and noggin, and enhancement of osteogenic potential. Bone 36: 254-266, 2005.

19. Moerman EJ, Teng K, Lipschitz DA and Lecka-Czernik B: Aging activates adipogenic and suppresses osteogenic programs in mesenchymal marrow stroma/stem cells: the role of PPAR- $\gamma$ transcription factor and TGF- $\beta /$ BMP signaling pathways. Aging Cell 3: 379-389, 2004.

20. Beck GR Jr, Zerler B and Moran E: Phosphate is a specific signal for induction of osteopontin gene expression. Proc Natl Acad Sci USA 97: 8352-8357, 2000.

21. Pittenger MF, Mackay AM, Beck SC, et al: Multilineage potential of adult human mesenchymal stem cells. Science 284 143-147, 1999.
22. Wu J, Jin F, Tang L, et al: Dentin non-collagenous proteins (dNCPs) can stimulate dental follicle cells to differentiate into cementoblast lineages. Biol Cell 100: 291-302, 2008.

23. Zhu L and Skoultchi AI: Coordinating cell proliferation and differentiation. Curr Opin Genet Dev 11: 91-95, 2001.

24. Okada $\mathrm{H}$ and Murakami S: Cytokine expression in periodontal health and disease. Crit Rev Oral Biol Med 9: 248-266, 1998.

25. Roberts FA, McCaffery KA and Michalek SM: Profile of cytokine mRNA expression in chronic adult periodontitis. J Dent Res 76: 1833-1839, 1997.

26. Ioannidou E: Therapeutic modulation of growth factors and cytokines in regenerative medicine. Curr Pharm Des 12: 2397-2408, 2006. 